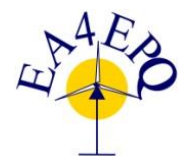

International Conference on Renewable Energies and Power Quality (ICREPQ'16)

Madrid (Spain), $4^{\text {th }}$ to $6^{\text {th }}$ May, 2016

Renewable Energy and Pourer Quality. Fournal (RE\&PQJ)

ISSN 2172-038 X, No.14 May 2016

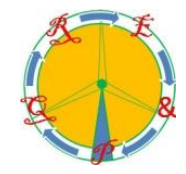

\title{
Cooperative Control Strategy of Multifunctional Inverters For Power Quality Enhancement in Smart Microgrid
}

\author{
C. Stevanoni, O. Deblecker and F. Vallée \\ Electrical Engineering Department \\ Faculty of Engineering, University of Mons (UMONS) \\ Boulevard Dolez 31, 7000 Mons (Belgium) \\ Phone:+0032 65 374114, e-mail: charline.stevanoni@umons.ac.be
}

\begin{abstract}
This paper deals with a cooperative strategy, based on the conservative power theory decomposition, which can be applied to control the inverters used to connect the distributed energy resources in a three-phase microgrid. In addition to inject/absorb active power at their point of connection, these inverters are operated according to their available power capability so as to enhance the power quality at the point of common coupling to the main network. Simulation results are shown considering, as an example, a three-phase three-wire microgrid equipped with two inverters, together with unbalanced and nonlinear loads. Among the different analyzed situations, the possibility to supply reactive power at the point of common coupling is shown with a view to provide voltage support, which is an asset in the framework of smart distribution networks.
\end{abstract}

Keywords. Power quality, Conservative Power Theory (CPT), cooperative control, multifunctional inverters, voltage support.

\section{Introduction}

During the last years, low-voltage power distribution networks have been subject to important changes because of the proliferation of distributed microgeneration of electric energy [1]. This fact has been driving the integration of a large amount of information and communication technologies into these networks. Among the many different aspects of this transition, this work deals with a control approach, based on the conservative power theory (CPT) decomposition [2], to enhance the power quality inside a smart microgrid (MG). A MG is a part of a low-voltage distribution network that is managed autonomously from the rest of the network in order to achieve better quality of service and increase distribution efficiency [3], [4]. Together with the connected loads, a MG includes renewable energy sources (solar panels, wind turbines, etc.) and energy storage systems. The latter are used to supply more flexibility to the network, providing a back-up to intermittent renewable energy. Those distributed energy resources (DERs) are connected to the MG by means of power electronic converters (switchmode inverters), whose main task is to control the active power flow exchanged with the network. However, they can also perform extra services to provide to the network performances like ride-through capability, power quality enhancement, etc. [5].

Indeed, the inverters of the microgeneration units are generally oversized, because most of the renewable energy sources are intermittent in time, and the switching power converters are designed according to their peak power production. When they are not working at the rated power, these inverters can therefore be commanded to provide additional tasks.

The coordinated control strategy of inverters related to DERs using the CPT has recently been explored in [6]. This theory has also been used for active shunt compensation to provide reactive compensation and harmonic filtering [7]. In a more global vision, those inverters are said multifunctional and different topologies and control features of them are presented in [8] and [9]. Following the works in [10], [11], the main contributions of this paper are as follows. First, we investigate the possibility of controlling the multifunctional inverters in a cooperative fashion from a central controller, in order to either fully or partially compensate the load unbalance, reactive power and harmonic distortion in a three-phase three-wire MG. Such a strategy simultaneously allows the inverters to locally inject/absorb active power into/from the $\mathrm{MG}$, as well as to work for improving the power quality at the point of common coupling (PCC) to the main network. The MG is indeed operated in gridconnected mode, being partially supplied from the main network, for reasons of availability, operations/stability and economics [1]. Second, based on the CPT definitions, an original method to adjust the reactive power exchanged with the main network is exposed with a view to provide voltage support.

The remainder of this paper is organized as follows. In Section 2, the CPT is briefly reviewed. Then, in Section 3 , the cooperative control strategy based on the CPT decomposition is exposed, together with the design of the current controller used to achieve the desired currents commands addressed to the inverters. Finally, simulation results are presented in Section 4 in order to validate the implemented strategy under different scenarios. 


\section{Review of the conservative power theory}

The CPT is defined in the time domain, for general operating conditions, and can be applied to single- and polyphase systems, with or without neutral wire. The theory proposes an orthogonal decomposition of currents and powers in the stationary frame, according to terms which are directly related to specific physical phenomena such as average power transfer, reactive energy, unbalance and distortion [2].

Consider a $M$-phase network under periodic operation (period $T$ ), where $\underline{u}, \underline{i}$ and $\underline{\hat{u}}$ are, respectively, the $M$ dimensional vectors of phase voltages, currents and unbiased voltage time integrals (i.e. AC components of the phase voltage integrals) measured at a generic network node. According to the CPT, the instantaneous power $(p)$ and the instantaneous reactive energy $(w)$ are conservative quantities, independently of the voltage and current waveforms:

$$
p=\underline{u} \cdot \underline{i}, \quad w=\underline{\hat{u}} \cdot \underline{i}
$$

The corresponding average active power $\left(P_{\mu}\right)$ and average reactive energy $\left(W_{\mu}\right)$ in each phase $\mu$ are defined as follows:

$$
\begin{gathered}
P_{\mu}=\left\langle u_{\mu}, i_{\mu}\right\rangle=\frac{1}{T} \int_{0}^{T} u_{\mu} i_{\mu} d t \quad \Rightarrow \quad P=\sum_{\mu=1}^{M} P_{\mu} \\
W_{\mu}=\left\langle\hat{u}_{\mu}, i_{\mu}\right\rangle=\frac{1}{T} \int_{0}^{T} \hat{u}_{\mu} i_{\mu} d t \quad \Rightarrow \quad W=\sum_{\mu=1}^{M} W_{\mu}
\end{gathered}
$$

Further, using the CPT, the current $i_{\mu}$ in each phase is decomposed according to its active and reactive components.

The active current $i_{a \mu}$ is the minimum phase current needed to convey active power $P_{\mu}$. It can be shown that such current has no impact on the reactive energy. It is expressed by:

$$
\begin{gathered}
i_{a \mu}=\frac{\left\langle u_{\mu}, i_{\mu}\right\rangle}{\left\|u_{\mu}\right\|^{2}} u_{\mu}=\frac{P_{\mu}}{U_{\mu}^{2}} u_{\mu}=G_{\mu} u_{\mu} \\
\Rightarrow \quad \mathbf{I}_{a}=\sqrt{\sum_{\mu=1}^{M} I_{a \mu}^{2}}=\sqrt{\sum_{\mu=1}^{M}\left(\frac{P_{\mu}}{U_{\mu}}\right)^{2}}
\end{gathered}
$$

where $G_{\mu}$ represents the equivalent conductance of phase $\mu$ and $\left\|u_{\mu}\right\|\left(=U_{\mu}\right)$ is the norm (rms value) of the phase voltage. Bold notation (here, e.g., $\mathbf{I}_{a}$ ) refers to the collective rms value.

On the other hand, the reactive current $i_{r \mu}$ is the minimum phase current needed to convey reactive energy $W_{\mu}$. It is expressed by:

$$
\begin{aligned}
& i_{r \mu}=\frac{\left\langle\hat{u}_{\mu}, i_{\mu}\right\rangle}{\left\|\hat{u}_{\mu}\right\|^{2}} \hat{u}_{\mu}=\frac{W_{\mu}}{\hat{U}_{\mu}^{2}} \hat{u}_{\mu}=B_{\mu} \hat{u}_{\mu} \\
& \Rightarrow \quad \mathbf{I}_{r}=\sqrt{\sum_{\mu=1}^{M} I_{r \mu}^{2}}=\sqrt{\sum_{\mu=1}^{M}\left(\frac{W_{\mu}}{\hat{U}_{\mu}}\right)^{2}}
\end{aligned}
$$

where $B_{\mu}$ is the equivalent reactivity of phase $\mu$. The reactive current has no influence on the active power.

Hence, it remains a residual term, called void current $i_{v \mu}$, neither linked to $P_{\mu}$ nor to $W_{\mu}$, defined by:

$$
i_{v \mu}=i_{\mu}-i_{a \mu}-i_{r \mu}
$$

All the aforementioned current terms are orthogonal (decoupled) to each other. Then:

$$
\begin{aligned}
& I_{\mu}=\left\|i_{\mu}\right\|=\sqrt{I_{a \mu}^{2}+I_{r \mu}^{2}+I_{v \mu}^{2}} \\
& \Rightarrow \quad \mathbf{I}=\sqrt{\mathbf{I}_{a}^{2}+\mathbf{I}_{r}^{2}+\mathbf{I}_{v}^{2}}
\end{aligned}
$$

To identify the effects of load unbalance and supply voltage asymmetry, the current terms can also be decomposed into balanced and unbalanced components. For instance, the $M$-dimensional vectors of balanced active and reactive currents, denoted $\underline{i}_{a}^{b}$ and $\underline{i}_{r}^{b}$ respectively, are defined as the minimum currents needed to convey the total active power $P$ and total reactive energy $W$ at the given node:

$$
\begin{aligned}
& \underline{i}_{a}^{b}=\frac{P}{\sum_{\mu=1}^{M} U_{\mu}^{2}}=\frac{P}{\mathbf{U}^{2}} \underline{u}=G^{b} \underline{u} \Rightarrow \mathbf{I}_{a}^{b}=\frac{P}{\mathbf{U}} \\
& \underline{i}_{r}^{b}=\frac{W}{\sum_{\mu=1}^{M} \hat{U}_{\mu}^{2}}=\frac{W}{\hat{\mathbf{U}}^{2}} \underline{\hat{u}}=B^{b} \underline{\hat{u}} \Rightarrow \mathbf{I}_{r}^{b}=\frac{W}{\hat{\mathbf{U}}}
\end{aligned}
$$

where $G^{b}$ and $B^{b}$ are, respectively, the equivalent balanced conductance and the equivalent balanced reactivity.

The unbalanced active and reactive currents in each phase $\mu$ at the given node are defined by difference as follows:

$$
i_{a \mu}^{u}=\left(G_{\mu}-G^{b}\right) u_{\mu}, \quad i_{r \mu}^{u}=\left(B_{\mu}-B^{b}\right) \hat{u}_{\mu}
$$

The balanced and unbalanced current terms are orthogonal to each other. Thus:

$$
\begin{aligned}
& \sum_{\mu=1}^{M}\left\langle i_{a \mu}^{u}, i_{a \mu}^{b}\right\rangle=0 \Rightarrow \mathbf{I}_{a}^{u}=\sqrt{\mathbf{I}_{a}^{2}-\mathbf{I}_{a}^{b 2}} \\
& \sum_{\mu=1}^{M}\left\langle i_{r \mu}^{u}, i_{r \mu}^{b}\right\rangle=0 \Rightarrow \mathbf{I}_{r}^{u}=\sqrt{\mathbf{I}_{r}^{2}-\mathbf{I}_{r}^{b 2}}
\end{aligned}
$$

and the global unbalanced phase currents are obtained by the sum of the unbalanced active and reactive components (which are orthogonal to each other as well):

$$
\underline{i}^{u}=\underline{i}_{a}^{u}+\underline{i}_{r}^{u} \Rightarrow \mathbf{I}^{u}=\sqrt{\mathbf{I}_{a}^{u 2}+\mathbf{I}_{r}^{u 2}}
$$

The last step is to decompose the apparent power $A$ introducing different power terms linked to the above defined current components:

$$
\begin{aligned}
A^{2} & =P^{2}+Q^{2}+N^{2}+V^{2} \\
& =\mathbf{U}^{2} \mathbf{I}_{a}^{b 2}+\mathbf{U}^{2} \mathbf{I}_{r}^{b 2}+\mathbf{U}^{2} \mathbf{I}^{u 2}+\mathbf{U}^{2} \mathbf{I}_{v}^{2}
\end{aligned}
$$

where $P$ is the active power, $Q$ the reactive power, $N$ the unbalance power and $V$ the void power.

Note that, from (9) and (14), the reactive power can be expressed in terms of reactive energy as follows:

$$
Q=\frac{\mathbf{U}}{\hat{\mathbf{U}}} W
$$

Finally, the power factor is defined as:

$$
P F=\frac{|P|}{A}
$$




\section{Cooperative control strategy using multifunctional inverters}

In this section, we present the cooperative control strategy, based on the CPT decomposition, using multifunctional inverters. The principle diagram of a multiple-inverterbased MG connected to the main network is shown in Fig. 1. As will be shown, in addition to the active power injection/absorption at the point of connection (POC) of each inverter, the implemented strategy allows for the improvement of power quality at the PCC.

Hereafter, the following assumptions are made: 1) the system is three-phase, three-wire (no zero sequence current terms), and 2) the power converters are two-level pulse width-modulation (PWM) inverters fed by ideal DC constant voltage sources. For simplicity, the LCL filter normally used to mitigate the harmonics generated by each inverter is replaced by a filter inductor.

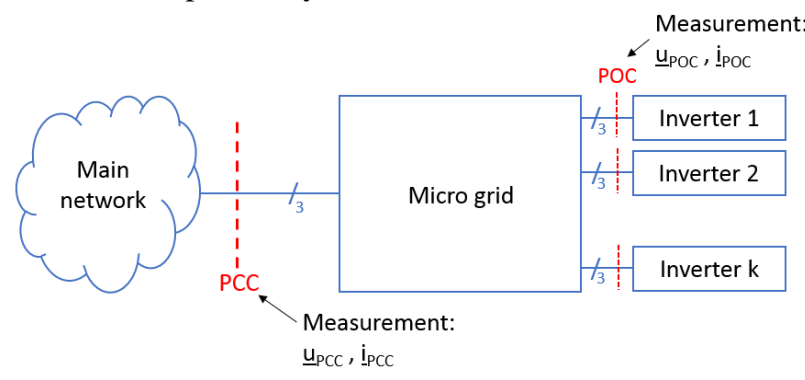

Fig. 1. Multiple-inverter-based MG connected to the main network.

The block diagram of the cooperative control strategy is shown in Fig. 2 for two inverters. The measurements needed consist of the voltages and currents at the PCC and POCs. The superscript " $p$ " refers to the fundamental positive-sequence.

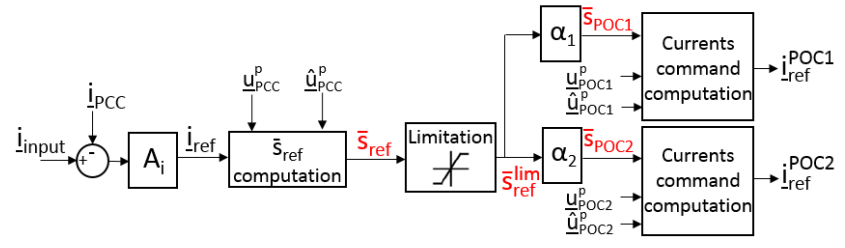

Fig. 2. Block diagram of the cooperative control strategy.

\section{A. Reference three-phase currents at the PCC}

Let us denote by $\underline{i}_{\text {input }}$, the three-phase instantaneous currents taken as references at the PCC. Comparing with the actual three-phase currents $\underline{i}_{P C C}$ absorbed at that node, the error signals vector (i.e. the currents to be compensated) is formed and amplified by a gain $A_{i}$ in order to generate the internal current references $\underline{i}_{r e f}$.

Using the power theory reviewed above, the instantaneous currents vector at the PCC can be decomposed into several terms as follows:

$$
\underline{i}_{P C C}=\underline{i}_{a}^{b}+\underline{i}_{r}^{b}+\underline{i}^{u}+\underline{i}_{v}
$$

Recall once more that all the terms are orthogonal to each other.

Thus, for example, if the effects linked to the reactive, unbalance and void powers at the PCC have to be compensated, the input vector $\underline{i}_{\text {input }}$ is put equal to the balanced active current term $\underline{i}_{a}^{b}$, which can be easily calculated from (8), provided that the total active power and instantaneous voltages $\underline{u}_{P C C}$ at the given node are known (such quantities can be measured by simple instrumentation). Hence, the error signals vector is given by $-\left(\underline{i}_{r}^{b}+\underline{i}^{u}+\underline{i}_{v}\right)$.

\section{B. Complex power reference, its limitation and partitioning}

Now, consider the instantaneous complex power defined from the instantaneous power and reactive energy (i.e. the conservative quantities in the CPT). The complex power reference at the $\mathrm{PCC}$ is written as:

$$
\bar{s}_{r e f}=p_{r e f}+j w_{r e f}
$$

with

$$
p_{r e f}=\underline{u}_{P C C}^{p} \cdot \underline{i}_{r e f}, \quad w_{r e f}=\underline{\hat{u}}_{P C C}^{p} \cdot \underline{i}_{r e f}
$$

As it can be noticed, the fundamental positive-sequence of the three-phase voltages and that of their unbiased time integral are used to evaluate the complex power in terms of the reference commands $p_{\text {ref }}$ and $w_{\text {ref. }}$. This can be justified from the fact that, unlike effects due to the loads into the microgrid, it would be unrealistic to use the inverters to compensate for the voltage asymmetry and/or distortion which are caused by the main network. Considering only fundamental positive-sequence in the definitions (19) provides, therefore, a mean to escape from the non-ideality of the voltage source.

The use of reference commands based on conservative quantities (see (19)) allows them to be partitioned and addressed to the different inverters from a central controller. Yet, before that, it is important to verify that the norm of the complex power reference does not exceed the total available compensation capability $A^{\text {tot }}$. These terms can be calculated respectively as follows:

$$
\begin{aligned}
& \left\|\bar{S}_{r e f}\right\|=S_{r e f}=\sqrt{p_{r e f}^{2}+w_{r e f}^{2}}, \\
& A^{t o t}=\sqrt{A_{P O C 1}^{2}+A_{P O C 2}^{2}}
\end{aligned}
$$

where $A_{P O C j}(j=1,2)$ is the apparent power of inverter $j$. From there, let us define the limited complex power reference as:

$$
\bar{s}_{r e f}^{\lim }=K \bar{s}_{r e f}
$$

where $K=A^{\text {tot }} / S_{\text {ref. }}$. If $S_{\text {ref }}$ becomes higher than $A^{\text {tot }}$, then $K<1$ and the output of the limitation bloc in Fig. 2 consists of the saturated reference commands. Otherwise, $K$ is taken equal to 1 (no limitation).

In this work, the reference commands are partitioned according to a criterion based on the power ratings of the inverters. For instance, considering two power converters rated for $A_{P O C 1}$ and $A_{P O C 2}$, we use the following weighting coefficients:

$$
\alpha_{1}=\frac{A_{P O C 1}}{A_{P O C 1}+A_{P O C 2}}, \quad \alpha_{2}=\frac{A_{P O C 2}}{A_{P O C 1}+A_{P O C 2}}
$$

whose sum is equal to 1 .

\section{Inverter reference three-phase currents}

At this stage, the reference commands (or complex power reference) addressed to each multifunctional inverter must be transformed into a three-phase instantaneous currents command. To that end, the relationships (19) must be rewritten by considering the phase voltages and currents at the point of connection of the given inverter $j$ : 


$$
p_{r e f}^{P O C}=\underline{u}_{P O C}^{p} \cdot \underline{i}_{r e f}^{P O C}, \quad w_{r e f}^{P O C}=\underline{\hat{u}}_{P O C}^{p} \cdot \underline{i}_{r e f}^{P O C}
$$

On the other hand, the zero-sequence currents at the POC must be null:

$$
\sum_{\mu=1}^{3} i_{\mu, r e f}^{P O C}=0
$$

and hence the sought command is obtained from a matrix inversion according to:

$$
\underline{i}_{r e f}^{P O C}=\left(\begin{array}{ccc}
u_{1} & u_{2} & u_{3} \\
\hat{u}_{1} & \hat{u}_{2} & \hat{u}_{3} \\
1 & 1 & 1
\end{array}\right)^{-1}\left(\begin{array}{c}
p_{r e f}^{P O C} \\
w_{r e f}^{P O C} \\
0
\end{array}\right)
$$

where, to lighten the notation:

$$
\left(u_{1}, u_{2}, u_{3}\right) \equiv \underline{u}_{P O C}^{p}, \quad\left(\hat{u}_{1}, \hat{u}_{2}, \hat{u}_{3}\right) \equiv \underline{\hat{u}}_{P O C}^{p}
$$

In order to execute the currents command, several control techniques are available in the literature (see, e.g., [12] for a review). Among them, resonant regulators can be used to control both voltage and current [13].

In this contribution, proportional-resonant (PR) regulators (from the fundamental up to eleventh harmonic) are implemented in parallel in the stationary frame, along with a modulator to create the duty cycles for the PWM pattern [14]. As an example, the current controller block diagram for phase 1 is shown in Fig. 3. $L_{1}$ represents the filter inductor per phase at the output of each inverter.

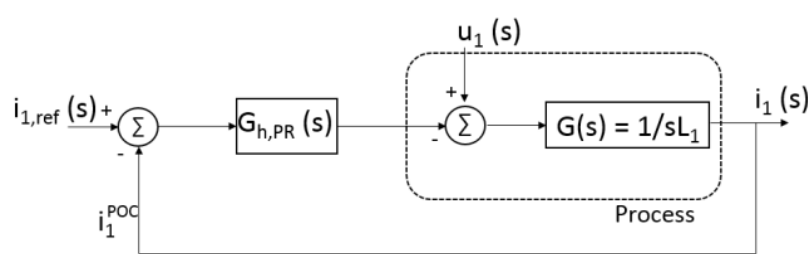

Fig. 3. Structure of the current control loop with PR regulators (in phase 1).

The design of the controller can be conducted as follows. Recall first that the transfer function of a PR regulator is given by:

$$
G_{h, P R}(s)=K_{p}+\sum_{h} \frac{K_{i} s}{s^{2}+\left(\omega_{0} \cdot h\right)^{2}}
$$

where $h$ is the harmonic order, $s$ the Laplace variable, $\omega_{0}$ $(=2 \pi / T)$ the fundamental angular frequency, $K_{p}$ the proportional gain and $K_{i}$ the integral gain.

The relationship between the input and the output of the current loop shown in Fig. 3 can be derived as:

$$
i_{1}(s)=H_{i}(s) i_{1, r e f}(s)+H_{u}(s) u_{1}(s)
$$

where

$$
\begin{aligned}
& H_{i}(s)=\frac{G_{h, P R}(s) G(s)}{G_{h, P R}(s) G(s)-1} \\
& H_{u}(s)=\frac{G(s)}{1-G_{h, P R}(s) G(s)}
\end{aligned}
$$

Since $G_{h, P R}(s)$ has an infinite gain at the resonant frequency $h \omega_{0}$, the $H_{u}(s)$ term in (27) can be neglected. Therefore, it is not necessary to have the voltage feedforward in the current control loop.
Finally, the gains are chosen to be $K_{p}=5$ and $K_{i}=2000$ for every PR regulator. With these values, the bode plots corresponding to the fundamental frequency as resonant term are shown in Fig. 4. The closed-loop transfer function $H_{i}(s)$ has a phase margin of about $60^{\circ}$.
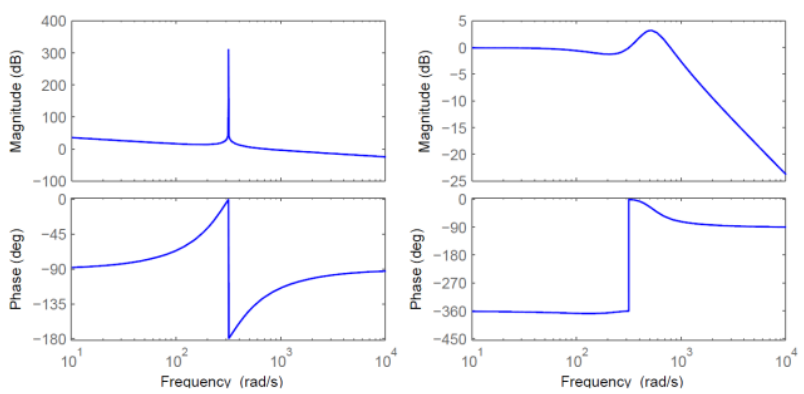

Fig. 4. Bode plots of $G_{h, P R}(s) G(s)$ (left) and $H_{i}(s)$ (right).

\section{Absorption/injection of active power by the inverters}

So far, it was supposed that the power capability of each inverter connected to the MG was fully available for compensation at the PCC. However, as previously mentioned, the main task of those inverters is to control locally the active power flow exchanged with the network.

Thus, by analogy with (8), for a given active power reference $P_{r e f}$ to be transferred by the inverter, a new active current term based on the CPT is defined as follows:

$$
\underline{i}_{a, P O C}^{b}=G_{P O C}^{b} \underline{u}_{P O C}
$$

with the equivalent balanced conductance:

$$
G_{P O C}^{b}=\frac{P_{r e f}}{\mathbf{U}_{P O C}^{2}}
$$

Then, the currents command discussed in Section 3-C now becomes the result of the addition of two terms, respectively given by (25) and (29).

Note that the sign of $P_{r e f}$ defines the direction of the active power flow.

It should also be noted that the currents $\underline{i}_{\text {input }}$ taken as references at the PCC (see Section 3-A) will change when the converters absorb/inject active power from/into the MG. Indeed, these are calculated by using (8) which shows a dependence with the total active power. Another consequence is the amount of apparent power available for compensation that becomes lower, given by:

$$
A_{P O C}^{\prime}=\sqrt{A_{P O C}^{2}-P_{r e f}^{2}}
$$

Hence, the weighting coefficients used to partition the reference commands (see (22)) should be calculated accordingly (i.e. using the new definition (31)).

\section{Application example}

We consider the $100 \mathrm{kVA}$ MG shown in Fig. 5, including unbalanced and distorting loads, a transformer, transmission lines, and two inverters rated 20 and 40 $\mathrm{kVA}$ respectively. This low-voltage network is inspired from reference [15]. The supply voltages fed at the PCC are $400 \mathrm{~V}, \quad 50 \mathrm{~Hz}$ (line-to-line, positive-sequence components), affected by an asymmetry of $2 \%$ (negative-sequence components). 
The proposed system was simulated by using a cosimulation with Simulink and PSIM softwares.

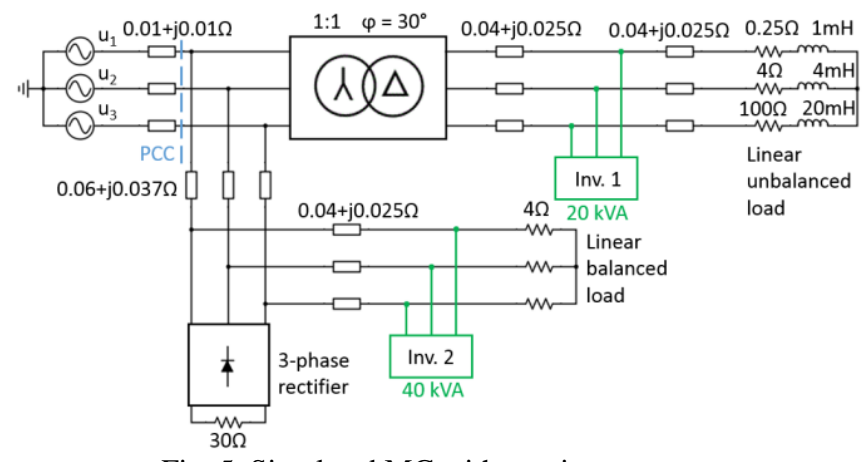

Fig. 5. Simulated MG with two inverters.

\section{A. Full compensation}

First, the cooperative control strategy is applied to compensate for the load unbalance, reactive power and harmonic distortion at the PCC (full compensation). It is supposed that the power capability of each inverter is fully available for compensation (i.e., for each, the active power reference is set to zero). The weighing coefficients $\alpha_{1}$ and $\alpha_{2}$ are chosen equal to $1 / 3$ and $2 / 3$, respectively.

The network operation is first analyzed without any type of compensation. At $t=0.7 \mathrm{~s}$, the cooperative control is activated with a unitary gain $A_{i}$. Then, at $t=1.4 \mathrm{~s}$, the gain is increased to 12 so as to enhance the compensation effect. The simulation results are shown in Fig. 6 and 7.

As can be seen in Fig. 6, the unbalance $(N)$, reactive $(Q)$ and void $(V)$ powers at the PCC are significantly reduced under the compensating action of the inverters, provided that the gain is sufficient. The corresponding time behaviours of the power factor $P F$ (given by (16)) and the unbalance factor $U F$ (defined as the ratio between the negative- and positive-sequence components of the currents at the PCC) are represented in Fig. 7. The expected values (near to 1 and zero, respectively) are reached after $t=1.4 \mathrm{~s}$, which reflects the improvement in terms of power quality. The total harmonic distortion $\left(T H D_{i}\right)$ of the three-phase currents at the PCC is reported in Table 1 at the different steps. The time waveforms of these currents, without and with compensation (taking $A_{i}=12$ ), are also shown in Fig. 8, allowing for a qualitative appreciation.

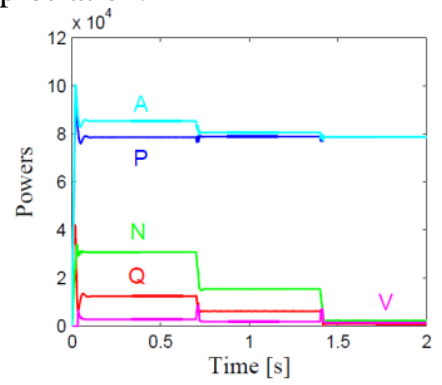

Fig. 6. Time behaviour of the power terms at the PCC.

\section{B. Injection of active power by the inverters}

In this scenario, we consider the injection of active power by the inverters at $t=0.5 \mathrm{~s}$. The cooperative control is simultaneously applied at $t=1 \mathrm{~s}$ to compensate for all the undesirable effects at the PCC (the gain $A_{i}$ is successively equal to 1 and 12 as in the previous case).
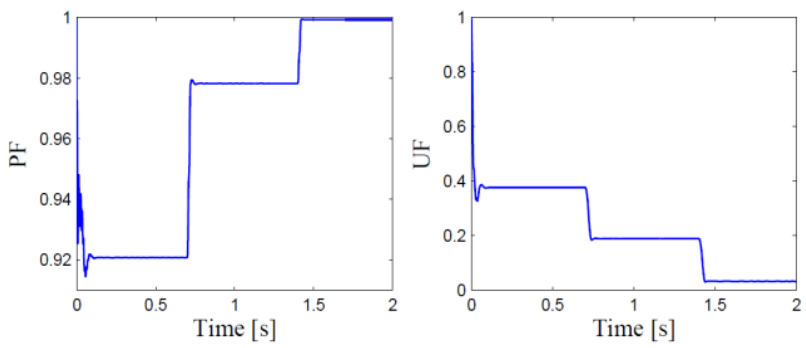

Fig. 7. Time behaviour of the power factor (left) and unbalance factor (right) at the PCC.
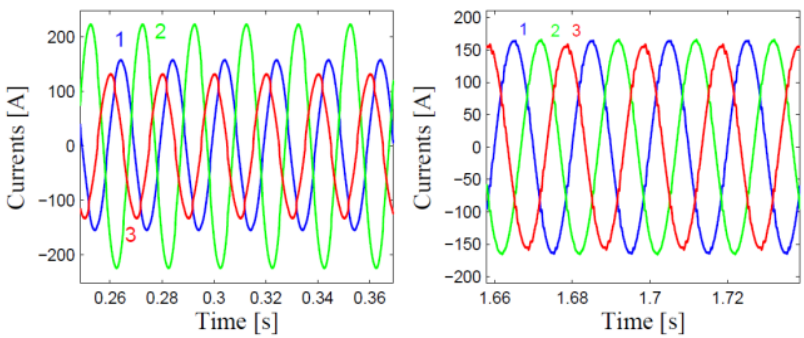

Fig. 8. Time waveforms of the currents at the PCC, without (left) and with compensation (right).

\begin{tabular}{|l|c|c|c|}
\hline & Phase 1 & Phase 2 & Phase 3 \\
\hline $\begin{array}{l}\text { Without } \\
\text { compensation }\end{array}$ & $3.49 \%$ & $2.47 \%$ & $4.69 \%$ \\
\hline With $A_{i}=1$ & $1.89 \%$ & $1.56 \%$ & $2.12 \%$ \\
\hline With $A_{i}=12$ & $1.43 \%$ & $1.63 \%$ & $1.86 \%$ \\
\hline
\end{tabular}

Table 1. Total harmonic distortion of the currents at the PCC.

Fig. 9 (left) shows the time behaviour of the different power terms. For the inverters 1 and 2, we apply a step of active power reference $P_{\text {ref }}$ from zero to $5 \mathrm{~kW}$ and from zero to $15 \mathrm{~kW}$, respectively. Also, referring to (31), the weighing coefficients $\alpha_{1}$ and $\alpha_{2}$ are now equal to 0.343 and 0.657. After a transient of about two fundamental time periods, this yields a reduction of $20 \mathrm{~kW}(=5+15 \mathrm{~kW})$ at the PCC (see Fig. 9 (right) for a zoom). The other power terms are not affected in steady state thanks to the decoupling inherent in the CPT decomposition. The power quality is clearly improved at the PCC, once the compensation operates.

Note also that, changing the sign of $P_{r e f}$, the absorption of active power by the multifunctional inverters can be analyzed in a similar way.
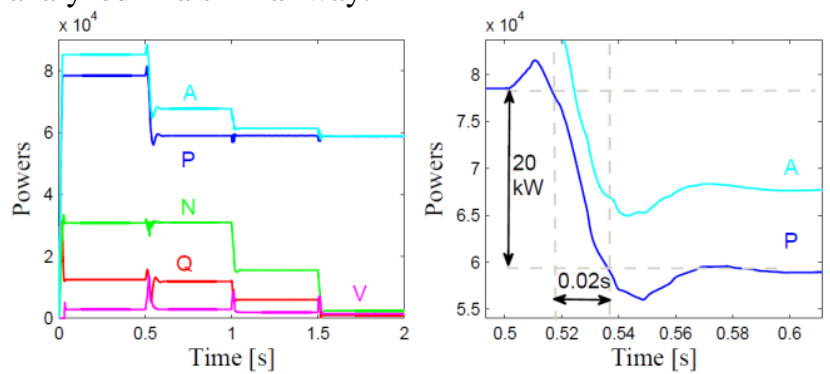

Fig. 9. Injection of $20 \mathrm{~kW}$ by the inverters, plus full compensation (left). Zoom on the response to the step of active power reference at $0.5 \mathrm{~s}$ (right).

\section{Supply/absorption of reactive power at the PCC}

Finally, with a view to provide voltage support to the main distribution system, the possibility of using the cooperative control strategy to supply or absorb a given amount of reactive power $\left(Q_{r e f}\right)$ at the PCC is shown. To 
that end, the input currents vector is put equal to both the balanced active and reactive current terms as follows:

$$
\underline{i}_{\text {input }}=\underline{i}_{a}^{b}+B_{P C C}^{b} \underline{\hat{u}}_{P C C}
$$

where from (9) and (15):

$$
B_{P C C}^{b}=\frac{W_{r e f}}{\hat{\mathbf{U}}_{P C C}^{2}}=\frac{Q_{r e f}}{\mathbf{U}_{P C C} \hat{\mathbf{U}}_{P C C}}
$$

The sign of $Q_{\text {ref }}$ is positive or negative, according to the direction of the reactive power flow (absorption from or injection into the main network, respectively). In principle, this amounts to under- or overcompensating the reactive power term at the PCC, respectively.

For the purpose of illustration, we consider the situation when the cooperative control is applied at $t=0.7 \mathrm{~s}$ with a gain $A_{i}=12$ and $Q_{r e f}=0$. The active power reference is set to zero for each inverter during the entire simulation time interval. At $t=1.4 \mathrm{~s}$, a (negative) step of reactive power reference $Q_{\text {ref }}$ is applied, from zero to $-8 \mathrm{kVAR}$.

The time behaviour of the different power terms is represented in Fig. 10 (left). The reactive power at the PCC diminishes from +10.9 kVAR initially to approximately $-6.4 \mathrm{kVAR}$ after $1.4 \mathrm{~s}$. It is worth noting that in order to supply the exact amount of reactive power (i.e. $-8 \mathrm{kVAR}$ ) to the main network, an additional outer control loop would be necessary to adjust the balanced reactive currents at the PCC. A zoom is shown in Fig. 10 (right) in order to visualize the transient (about one fundamental period). The unbalance and void powers are close to zero from the moment the control is activated.
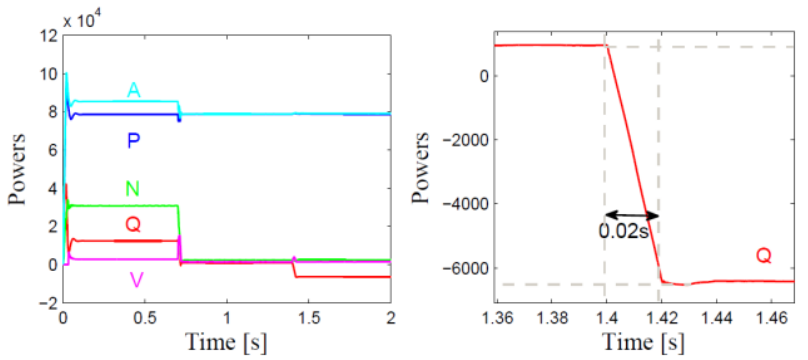

Fig. 10. Supply of $8 \mathrm{kVAR}$ to the main network, compensation of the unbalance and void powers (left). Zoom on the response to the step of reactive power reference at $1.4 \mathrm{~s}$ (right).

\section{Conclusion}

In this paper, a cooperative control strategy from a central controller has been implemented, which applies to the inverters used to connect the DERs in a three-phase MG. Such a strategy, simultaneously allows the inverters to exchange active power with the network at their point of connection, as well as to work for improving the power quality at the PCC to the main network. The CPT, defined for general operating conditions, was proved to offer a consistent framework to approach such control problem. Indeed, it proposes an orthogonal decomposition of currents and powers in the stationary frame, according to terms which are directly related to specific physical phenomena (average power transfer, unbalance, etc.). Hence, each power term can be compensated independently from the others. As an application example, a three-phase MG including unbalanced and nonlinear loads, together with two inverters, was simulated and the cooperative control strategy applied in different situations (full compensation, injection of active power by the inverters, etc.). It has been shown also that, defining adequately the balanced reactive current term at the PCC, the supply (or absorption) of reactive power to the main distribution network could be adjusted easily to provide voltage support, which is an original contribution. Future work will focus on more in-depth evaluation of this issue taking into account the model of the distribution feeder and placement of the MG within the main distribution system.

\section{References}

[1] M. Liserre, T. Sauter, and J. Y. Hung, "Future energy systems," IEEE Ind. Electron. Mag., pp. 18-37, Mar. 2010.

[2] P. Tenti, H. K. Paredes, and P. Mattavelli, "Conservative power theory, a framework to approach control and accountability issues in smart microgrids," IEEE Trans. on Power Electron., vol. 26, no. 3, Mar. 2011.

[3] E. Santacana, et al., "Getting smart with a clearer vision of the intelligent grid, control emerges from chaos," IEEE Power Energy Mag., vol. 8, no. 2, pp. 41-48, Mar. 2010.

[4] A. Ipakchi and F. Albuyeh, "Grid of the future, are we ready to transition to a smart grid," IEEE Power Energy Mag., vol. 7, no. 2, pp. 52-62, Mar. 2009

[5] M. Prodanovic, et al., "Harmonic and reactive power compensation as ancilliary services in inverter-based distribution generation," IET Generation, Transmission and Distribution, vol. 1, no. 3, pp. 432-438, 2007.

[6] A. Mortizaei, et al., "Coordinated operation in a multiinverter based microgrid for both grid-connectd and islanded modes using conservative power theory", in Proc. Energy Conversion Congress and Exposition (ECCE), Montreal, QC, pp. 4602-4609, Sep. 2015.

[7] T. S. Haugan and E. Tedeschi, "Reactive and harmonic compensation using the conservative power theory", in Proc. Tenth International Conference on Ecological Vehicles and renewable energies (EVER), 2015.

[8] A. Teke and M. B. Latran, "Review of multifunctional inverter topologies and control schemes used in distributed generation systems", Journal of Power Electronics, vol. 14, no. 2, pp. 324-340, March 2014.

[9] Z. Zeng, et al., "Objective-oriented power quality compensation of multifunctional grid-tied inverters and its application in microgrids", IEEE Trans. On Power Electronics, vol. 30, no. 3, pp. 1255-1265, March 2015.

[10] P. Tenti, et al., "Compensation of load unbalance, reactive power and harmonic distortion by cooperative operation of distributed compensators," in Proc. 13th Eur. Conf. Power Electron. and Appl., Barcelona, Spain, Sep. 2009.

[11] D. I. Brandão, et al., "Inverter control strategy for DG systems bases on the conservative power theory," in Proc. Energy Conversion Congress and Expo. (ECCE), Denver, USA, pp. 3283-3290, Sep. 2013.

[12] M. Hojabri, et al., "An overview on current control techniques for grid connected renewable energy systems," in Proc. 2nd Int. Conf. Power and Energy Sys., pp. 119-126, 2012. [14] M. Ebad and B.-M. Song, "Improved design and control of proportional resonant controller for three-phase voltage Source Inverter," in Proc. Power Electron. and Machines in Wind Appl. (PEMWA), Denver, USA, Jul. 2012.

[13] J. He, et al., "Flexible microgrid power quality enhancement using adaptive hybrid voltage and current controller", IEEE Trans. On Industrial Electronics, vol. 61, no.6, pp. 2784-2794, June 2014.

[15] A. Costabeber, et al., "Selective compensation of reactive, unbalance and distortion power in smart grids by synergistic control of distributed switching power interfaces", in Proc. EPE'13 ECCE Europe, Lille, France, Sep. 2013. 